\title{
Assistência de enfermagem ao paciente vítima de traumatismo cranioencefálico
}

\author{
Nursing assistance to the patient victim of cranioencephalic traumatism \\ Assistencia de enfermagem al paciente víctima de traumatismo craniencefálico
}

Recebido: 04/07/2021 | Revisado: 09/07/2021 | Aceito: 12/07/2021 | Publicado: 23/07/2021

João Felipe Tinto Silva

ORCID: https://orcid.org/0000-0003-3662-6673 Centro Universitário de Ciências e Tecnologia do Maranhão, Brasil E-mail: felipetinto99@gmail.com

Alaine dos Santos Silva Martins

ORCID: https://orcid.org/0000-0002-1898-4920 Universidade Estadual de Ciências da Saúde de Alagoas, Brasil E-mail: alaine.martins22@gmail.com Michelle Quaresma Cardoso

ORCID: https://orcid.org/0000-0002-8621-8670 Universidade Federal do Pará, Brasil E-mail: michelle.card91@ yahoo.com.br Manoel dos Santos Carvalho

ORCID: https://orcid.org/0000-0001-6762-3091 Universidade Federal do Piauí, Brasil

E-mail: mmano8791@ gmail.com

Jhônata Santos Brito

ORCID: https://orcid.org/0000-0002-8161-5677

Faculdade de Ciências e Empreendedorismo, Brasil

E-mail: jhonbrito12@gmail.com

Silvio Matheus Azevedo Rocha

ORCID: https://orcid.org/0000-0003-2579-4989

Universidade Tiradentes, Brasil

E-mail: silviomatheus@icloud.com

Vitaliano de Oliveira Leite Júnior

ORCID: https://orcid.org/0000-0001-5812-0150

Universidade Federal do Maranhão, Brasil

E-mail: vitalianojunior@gmail.com

Emmanoela de Almeida Paulino Lima

ORCID: https://orcid.org/0000-0002-7328-680X

Universidade Federal da Paraíba, Brasil

E-mail: manupaulino@gmail.com

Isabelly Raiane Silva dos Santos

ORCID: https://orcid.org/0000-0002-4034-6677 Universidade Federal do Pará, Brasil

E-mail: isabelly.santos@ifpa.edu.br

Samuel Carlos Tomaz

ORCID: https://orcid.org/0000-0003-3885-8857

Universidade Regional do Cariri, Brasi E-mail: samueltomaz47@yahoo.com

Raiane Lira dos Santos

ORCID: https://orcid.org/0000-0002-8278-4560

Centro Universitário do Estado do Pará, Brasil

E-mail: raianeliradossantos@gmail.com

Moacir Andrade Ribeiro Filho

ORCID: https://orcid.org/0000-0003-1991-469X Universidade Regional do Cariri, Brasi

E-mail: moacirarf@outlook.com

Jaqueline Brito da Costa

ORCID: https://orcid.org/0000-0002-2570-1714

Centro Universitário de Ciências e Tecnologia do Maranhão, Brasil

E-mail: jaquelinebritto2012@gmail.com

Bruno Abilio da Silva Machado

ORCID: https://orcid.org/0000-0003-1759-0206

Centro Universitário Maurício de Nassau, Brasil

E-mail: brunnoabilio92@gmail.com

Vitória Vilas Boas da Silva Bomfim

ORCID: https://orcid.org/0000-0003-4897-0279

Centro Universitário Jorge Amado, Brasi E-mail: pesquisaclinica9@gmail.com 


\begin{abstract}
Resumo
O presente estudo tem como objetivo realizar uma revisão integrativa acerca da assistência de enfermagem ao paciente vítima de traumatismo cranioencefálico. Trata-se de uma revisão integrativa da literatura, a busca ocorreu na Biblioteca Virtual em Saúde (BVS), com o auxílio das bases de dados Literatura Latino-Americana e do Caribe em Ciências da Saúde (LILACS), Bases de Dados de Enfermagem (BEDENF), Biblioteca Virtual Scientific Eletronic Library Online (SciELO) e PUBMED. Os artigos foram coletados no período de junho 2021. Foram utilizados os descritores: Traumatismos craniocerebrais, Assistência de enfermagem e Enfermagem. Após analises dos artigos encontrados, 10 estudos foram selecionados para compor o presente trabalho. O estudo aponta que o enfermeiro tem um papel fundamental no cuidado oferecido as vítimas de traumatismo cranioencefálico, sendo necessário que ele esteja apto para obter uma breve história do paciente, realize o exame físico, e execute o tratamento imediato, preocupando-se com a manutenção da vida. Deve aliar sua fundamentação teórica à capacidade de liderança, iniciativa e habilidades assistenciais e de ensino.
\end{abstract}

Palavras-chave: Traumatismo craniocerebrais; Assistência de enfermagem; Enfermagem.

\begin{abstract}
This study aims to carry out an integrative review of nursing care for patients who are victims of traumatic brain injury. This is an integrative literature review, the search took place in the Virtual Health Library (VHL), with the aid of the Latin American and Caribbean Literature in Health Sciences (LILACS) databases, Nursing Databases (BEDENF), Virtual Scientific Electronic Library Online (SciELO) and PUBMED. The articles were collected in the period of June 2021. The descriptors were used: Craniocerebral trauma, Nursing care and Nursing. After analyzing the articles found, 10 studies were selected to compose the present work. The study points out that the nurse has a fundamental role in the care offered to victims of traumatic brain injury, it's necessary for him to be able to obtain a brief history of the patient, perform the physical examination, and carry out immediate treatment, worrying about the maintenance of life. It must combine its theoretical foundation with leadership capacity, initiative and care and teaching skills. he needs to be quick thinking, as he is responsible for coordinating a nursing team, being a vital and integral part of the emergency team.
\end{abstract}

Keywords: Craniocerebral trauma; Nursing care; Nursing.

\begin{abstract}
Resumen
El objetivo de este estudio es realizar una revisión integradora de la atención de enfermería a los pacientes víctima de traumatismo craneoencefálico.. Se trata de una revisión integradora de la literatura, la búsqueda se realizó en la Biblioteca Virtual en Salud (BVS), con la ayuda de las bases de datos de Literatura Latinoamericana y del Caribe en Ciencias de la Salud (LILACS), Bases de Datos de Enfermería (BEDENF), Biblioteca Virtual Científica Electrónica en Línea ( SciELO) y PUBMED. Los artículos fueron recolectados en el período de junio de 2021. Se utilizaron los descriptores: Traumatismo craneoencefálico, Atención de enfermería y Enfermería. Tras analizar los artículos encontrados, se seleccionaron 10 estudios para componer este trabajo. O estudo aponta que o enfermeiro tem um papel fundamental no cuidado oferecido as vítimas de traumatismo cranioencefálico, sendo necessário que ele esteja apto para obter uma breve história do paciente, realize o exame físico, e execute o tratamento imediato, preocupandose com a manutenção de la vida. Debe combinar su fundamento teórico con la capacidad de liderazgo, la iniciativa y el cuidado y la docencia. Debe pensar rápido, ya que es responsable de coordinar un equipo de enfermería, siendo una parte vital e integral del equipo de emergencia.
\end{abstract}

Palabras clave: Traumatismos craniocerebrais; Asistencia de enfermería; Enfermería.

\title{
1. Introdução
}

O Traumatismo cranioencefálico (TCE) resulta de uma agressão ao crânio, que afeta a massa encefálica, causado por uma ação externa, que pode ser de baixa ou alta intensidade, acarretando ou não em comprometimento funcional, da estrutura do crânio, couro cabeludo, encéfalo, vasos ou meninges, sendo este tipo de ocorrência como um dos responsáveis por uma alta taxa de mortalidade em todo o mundo (Silva, Pio, \& Maia, 2019).

Quanto à fisiopatologia, pode ser dividido em duas fases. A primeira corresponde à lesão cerebral, que se caracteriza por trauma tecidual e desregulação do fluxo sanguíneo encefálico e do seu metabolismo, neste caso tem-se uma isquemia tecidual, que ocorre devido um acumulo de ácido lático proveniente da glicose anaeróbia, o que leva a um aumento da permeabilidade da membrana celular e consequente edema tecidual. Na segunda fase, a cascata de eventos se inicia por uma despolarização terminal da membrana junto com a liberação excessiva de neurotransmissores excitatórios, que ativam receptores e abrem os canais de sódio e cálcio-dependentes (Silva, \& Filha, 2017). 
No Brasil, o TCE atinge 500.000 mil pessoas hospitalizadas por ano, com taxas de 14 a 30 óbitos a cada 100.000 mil pessoas. Estima-se que entre 75 a 100 mil pessoas morrem nas primeiras horas do trauma, e outras 70 a 90 mil pessoas ficarão com sequelas irreversíveis. Destes, destacam-se os homens que têm maior risco de se envolver em acidentes do que as mulheres (Rezer, Pereira, \& Fautino, 2020).

Consideram-se situações de emergência, quando o indivíduo apresenta condições, que exigem intervenções imediatas de uma equipe de saúde, pois qualquer retardamento no atendimento e na implementação de medidas terapêuticas aumentam, substancialmente, a gravidade do quadro, além de representar ameaça potencial à vida do usuário ou severas complicações da lesão. Devido a este fato, é importante verificar como ocorre o atendimento na unidade de emergência, com foco principal no trauma craniano. Nesse contexto, a vítima de trauma deve ser considerada como paciente prioritário no serviço de Emergência pela potencialidade de sua gravidade, pois pode ter suas funções vitais prejudicadas em um curto período de tempo (Werlang, Badke, Freitag, Silva, Federizzi, \& Ribeiro, 2017). Esta escala é utilizada universalmente para classificar o nível de lesão hemisférica cerebral e sua classificação tem relação com a gravidade e a possibilidade de óbito pela lesão (Santos, 2020).

Pacientes que sofrem de TCE necessitam de tomada de decisão e avaliação em tempo hábil, para que se identifique e trate lesões que podem ocasionar em morte (Oliveira, Soares, Noleto, Fontinele, Galvão, \& Souza, 2018). A gravidade do TCE é determinada utilizando-se a Escala de Coma de Glasgow (GCS), obtida pela observação de três parâmetros: abertura ocular, resposta verbal e resposta motora. Foi descrita em 1974 por Teasdale e Jennet, categorizando como grave uma pontuação de 3 a 8, moderado 9 a 12 e como leve 13 a 15 (Monteiro, Frasson, Wrsesinski, Bardini, Lin, \& Fernandes, 2016).

Entre os profissionais envolvidos no acolhimento e primeiros cuidados com esse perfil de vítima, destaca-se o enfermeiro, com assistência qualificada em suporte de vida. Essa assistência deve incluir comunicação favorável, imobilização e manutenção da respiração, hemodinâmica e nível de consciência, porém não se limita a aspectos clínicos. A liderança, habilidades específicas junto a pacientes graves e/ou com eventos clínicos mais complexos e a coordenação do cuidado seguro, consolidam as atribuições deste profissional junto à vítima com TCE (Rezer, pereira, \& Faustino, 2020).

A equipe de enfermagem tem um papel importante na assistência ao paciente vítima de TCE, tendo ainda como função realizar uma avaliação diagnóstica e terapêutica, com a finalidade de reanimação e estabilização das funções ventilatórias e hemodinâmica do paciente, investigando informações da história de saúde imediata, os mecanismos do trauma, o tipo de acidente ocorrido, avaliação da consciência da vítima após o traumatismo, tempo de duração, podendo indicar um grau significativo de comprometimento cerebral (Ribeiro, Freitas, Gregório, \& Silva, 2015).

Dessa forma, a função do enfermeiro torna-se essencial no cuidado oferecido a esses pacientes, havendo necessidade de maior aptidão para coletar uma breve história da vítima, realizar o exame físico e intervir através do tratamento imediato, visando minimizar as sequelas e salvar a vida do paciente, sendo este atendimento de qualidade e eficaz (Cunha, Araújo, \& Vieira, 2015; Fettermann, Aranda, \& Kist, 2018).

Neste contexto, o presente trabalho tem como objetivo realizar uma revisão integrativa acerca da assistência de enfermagem ao paciente vítima de traumatismo cranioencefálico.

\section{Metodologia}

Trata-se de uma pesquisa revisão integrativa da literatura. A revisão de literatura configura-se, portanto como um tipo de revisão que reúne achados de estudos desenvolvidos mediante de diferentes metodologias, permitindo aos revisores sintetizar resultados sem ferir a filiação epistemológica dos estudos empíricos incluídos. Para que esse processo se concretize de maneira lógica, isenta de desatinos epistemológicos, a revisão requer que os revisores procedam à análise e à síntese dos dados primários de maneira sistemática e rigorosa (Soares, Hoga, Peduzzi, Sangaleti, Yonekura, \& Silva, 2014). 
Para a revisão integrativa da literatura foram percorridas as seguintes etapas: identificação do tema e seleção da questão norteadora, estabelecimento de critérios de inclusão e exclusão de estudos, categorização e avaliação dos artigos incluídos na revisão, interpretação dos resultados e a síntese do conhecimento dos principais resultados evidenciados na análise dos artigos incluídos (Mendes, Silveira, \& Galvão, 2008).

O tema, determinou a construção da estratégia PICo, que representa uma acrômio para Paciente ou Problema (P), Interesse (I) e Contexto (Co), na qual foi utilizada para a geração da questão norteadora desta revisão integrativa da literatura: "Qual a assistência prestada pela equipe de enfermagem diante do paciente vítima de traumatismo cranioencefálico?".

Os estudos foram coletados no período de junho de 2021, realizada através da Biblioteca Virtual em Saúde (BVS), com auxílio das bases de dados Literatura Latino-Americana e do Caribe em Ciências da Saúde (LILACS), Base de Dados de Enfermagem (BDENF), Scientific Electronic Library Online (SciELO) e PUBMED.

Para a localização dos estudos relevantes, que respondessem à pergunta da pesquisa, utilizou-se os descritores: "Traumatismos craniocerebrais", "Assistência de Enfermagem" e "Enfermagem", localizados na lista dos Descritores em Ciências da Saúde (DeCS), disponíveis no portal da Biblioteca Virtual em Saúde em (http://decs.bvs.br/). Tais descritores foram cruzados utilizando os operadores booleanos "AND" para obtenção dos critérios de inclusão e exclusão.

Os critérios de inclusão estabelecidos para a seleção dos artigos foram: artigos completos publicados nas bases de dados elencadas, nos idiomas português, inglês e espanhol, com recorte temporal de 2014 a 2021 e relacionados com a temática. Foram excluídos cartas ao editor, resumos, resenhas, monografias, dissertações, teses, e artigos publicados em anais de eventos.

Foram identificados 522 artigos nas bases de dados, dos quais 17 estavam duplicados nas bases de dados, resultando em 505 artigos. Outros 437 foram excluídos após aplicação dos critérios de inclusão e exclusão, restando 68 artigos. Após a leitura dos títulos e resumos, apenas 17 artigos foram selecionados. Destes, após a leitura na integra, 11 artigos foram selecionados para compor a presente revisão, por responderem a pergunta norteadora da revisão. O fluxograma com o detalhamento das etapas de pesquisa está apresentado a seguir na Figura 1. 
Figura 1. Fluxograma de identificação e seleção dos artigos, 2021.

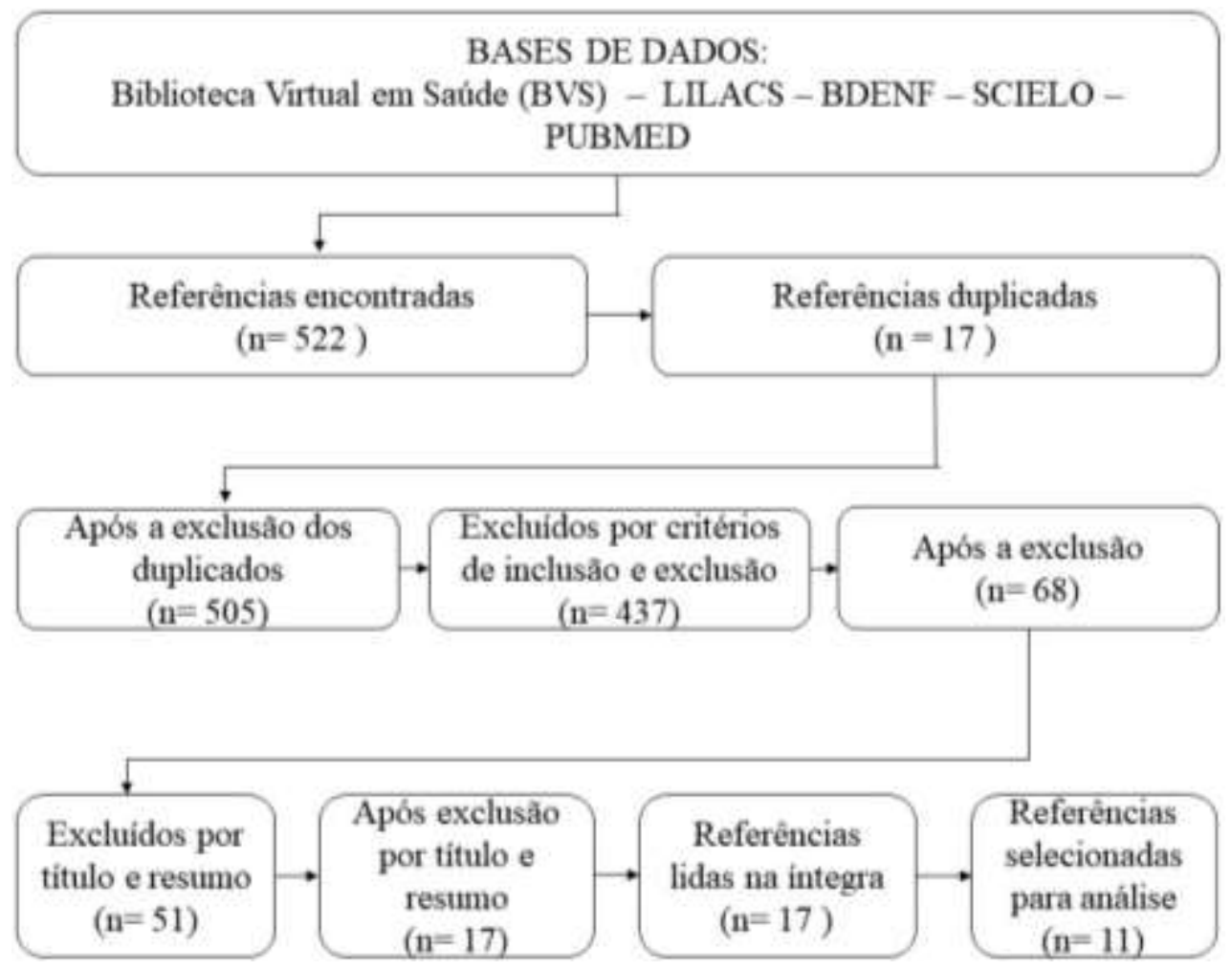

Fonte: Autores (2021).

\section{Resultados e Discussão}

Dos 11 artigos incluídos nesta revisão, 6 (54,5\%) estavam disponíveis na língua portuguêsa, 3 (27,3\%) na língua inglêsa e $2(18,2 \%)$ em espanhol. Destes, 1 (9,0\%)foi publicado em 2014, 1 (9,0\%) em 2018, 2 (18,2\%) em 2019, 2 (18,2\%) foi públicado em 2020, 2 (18,2\%) em 2015 E 3 (27,3\%) públicados em 2016.

O Quadro 1 apresenta os estudos selecionados para a composição da presente revisão integrativa da literatura de forma mais detalhada, seguido as informações como título do artigo, autores e ano de publicação, objetivo, metodologia e principais resultados.

Quadro 1. Distribuição dos artigos selecionados de acordo com o título, autores e ano de publicação, objetivos, metodologia e principais resultados, 2021.

\begin{tabular}{|c|c|c|c|c|}
\hline Título & Autores/Ano & Objetivo & Metodologia & Principais resultados \\
\hline $\begin{array}{l}\text { Trauma cranioencefálico: } \\
\text { perfil dos pacientes } \\
\text { atendidos em um hospital } \\
\text { público de Teresina }\end{array}$ & $\begin{array}{l}\text { Carvalho, Silva, } \\
\text { Viana, Madeira, } \\
\text { Oliveira, e } \\
\text { Carvalho 2020) }\end{array}$ & $\begin{array}{l}\text { Identificar o perfil dos pacientes } \\
\text { acometidos com traumatismo } \\
\text { cranioencefálico atendidas em um } \\
\text { hospital público da cidade de } \\
\text { Teresina }\end{array}$ & $\begin{array}{l}\text { Estudo } \\
\text { descritivo, } \\
\text { exploratório, } \\
\text { retrospectivo, } \\
\text { documental } \\
\text { de abordagem } \\
\text { quantitativa }\end{array}$ & $\begin{array}{l}\text { Conclui-se que o perfil do paciente } \\
\text { acometido por trauma cranioencefálico, } \\
\text { no hospital estudado, é caracterizado } \\
\text { por um predomínio do sexo masculino } \\
\text { com uma faixa etária de } 19 \text { a } 29 \text { anos, } \\
\text { solteiros e com escolaridade de até o } \\
\text { ensino fundamental. }\end{array}$ \\
\hline $\begin{array}{l}\text { Escalas para avaliação do } \\
\text { nível de consciência em } \\
\text { trauma cranioencefálico e } \\
\text { sua relevância para a } \\
\text { prática de enfermagem } \\
\text { em neurocirurgia }\end{array}$ & $\begin{array}{c}\text { Oliveira, Pereira } \\
\text { e Freitas } \\
(2014)\end{array}$ & $\begin{array}{l}\text { realizar uma revisão da literatura } \\
\text { sobre escalas para avaliação do } \\
\text { nível de consciência em pacientes } \\
\text { com trauma cranioencefálico, } \\
\text { chamando a atenção para a sua } \\
\text { importância na prática de } \\
\text { enfermagem em neurocirurgia }\end{array}$ & $\begin{array}{l}\text { Revisão } \\
\text { integrativa da } \\
\text { literatura }\end{array}$ & $\begin{array}{l}\text { A literatura consultada revela que, } \\
\text { apesar de vários estudos destacarem a } \\
\text { importância do tema, a avaliação } \\
\text { neurológica com a utilização de outras } \\
\text { escalas não é prática rotineira nas } \\
\text { unidades de trauma. }\end{array}$ \\
\hline
\end{tabular}




\begin{tabular}{|c|c|c|c|c|}
\hline $\begin{array}{lr}\text { Intervenções } & \text { de } \\
\text { enfermagem } & \text { para } \\
\text { pacientes neurocríticos }\end{array}$ & $\begin{array}{l}\text { Caciano et al., } \\
\text { (2019) }\end{array}$ & $\begin{array}{c}\text { Identificar as intervenções de } \\
\text { Enfermagem para pacientes } \\
\text { neurocríticos em uma Unidade de } \\
\text { Terapia Intensiva }\end{array}$ & $\begin{array}{l}\text { Estudo } \\
\text { quantitativo, } \\
\text { descritivo, } \\
\text { transversal. }\end{array}$ & $\begin{array}{l}\text { Relacionam-se as principais } \\
\text { intervenções de Enfermagem realizadas } \\
\text { ao posicionamento neurológico, } \\
\text { monitorização neurológica, } \\
\text { monitorização dos sinais vitais e à } \\
\text { prevenção de lesão por pressão. } \\
\text { Acredita-se que os resultados deste } \\
\text { estudo trazem importantes contribuições } \\
\text { para o planejamento da assistência ao } \\
\text { paciente neurocrítico, contribuindo para } \\
\text { a prática baseada em evidências } \\
\text { científicas na Enfermagem. }\end{array}$ \\
\hline $\begin{array}{ll}\text { Conhecimento } & \text { de } \\
\text { enfermeiros } & \text { na } \\
\text { abordagem à vítima } & \text { de } \\
\text { traumatismo } & \\
\text { cranioencefálico } & \end{array}$ & $\begin{array}{l}\text { Rezer, Pereira e } \\
\text { Faustino } \\
\text { (2020) }\end{array}$ & $\begin{array}{l}\text { Verificar o conhecimento de } \\
\text { enfermeiros sobre o traumatismo } \\
\text { cranioencefálico e a Escala de } \\
\text { Coma de Glasgow. }\end{array}$ & $\begin{array}{l}\text { Estudo } \\
\text { exploratório } \\
\text { com } \\
\text { abordagem } \\
\text { quantitativa }\end{array}$ & $\begin{array}{l}\text { O estudo aponta a importância dos } \\
\text { enfermeiros se manterem atualizados no } \\
\text { atendimento às vítimas de traumatismo } \\
\text { cranioencefálico, além da necessidade } \\
\text { de educação permanente e } \\
\text { aperfeiçoamento da equipe, para } \\
\text { melhoria do atendimento e segurança do } \\
\text { paciente. }\end{array}$ \\
\hline $\begin{array}{l}\text { Accidente de tráfico: } \\
\text { análisis de los casos de } \\
\text { traumatismo } \\
\text { cranoencefálico }\end{array}$ & $\begin{array}{l}\text { Marinho, Santos, } \\
\text { Filho, Valença, } \\
\text { Santos, \& Júnior } \\
\text { (2019) }\end{array}$ & $\begin{array}{l}\text { Caracterizar os pacientes vítimas } \\
\text { de acidentes de trânsito internados } \\
\text { em um hospital geral da cidade de } \\
\text { Natal e o acidente de trânsito por } \\
\text { eles sofrido. }\end{array}$ & $\begin{array}{l}\text { Estudo } \\
\text { exploratório, } \\
\text { descritivo, } \\
\text { transversal, } \\
\text { retrospectivo, } \\
\text { com } \\
\text { abordagem } \\
\text { quantitativa }\end{array}$ & $\begin{array}{l}\text { O perfil das vítimas de acidentes de } \\
\text { trânsito com traumatismo } \\
\text { cranioencefálico é caracterizado por } \\
\text { indivíduos do sexo masculino e } \\
\text { jovens. O acidente prevalece no final de } \\
\text { semana, tendo a motocicleta como } \\
\text { principal culpada e a ingestão de álcool } \\
\text { contribui para o evento. }\end{array}$ \\
\hline $\begin{array}{l}\text { The management of } \\
\text { minor head trauma (GCS } \\
\text { 15-13) across a Trauma } \\
\text { Network }\end{array}$ & $\begin{array}{l}\text { Pulhrn, } \\
\text { Westmoreland e } \\
\text { McMahon } \\
\text { (2016) }\end{array}$ & $\begin{array}{l}\text { Determinar as variações no } \\
\text { manejo de pacientes com } \\
\text { traumatismo craniano leve em uma } \\
\text { Rede de Trauma. }\end{array}$ & $\begin{array}{l}\text { Estudo } \\
\text { descritivo } \\
\text { com } \\
\text { abordagem } \\
\text { qualitativa }\end{array}$ & $\begin{array}{l}\text { O manejo de pacientes com } \\
\text { traumatismo cranioencefálico menor em } \\
\text { unidades de trauma deve ser } \\
\text { padronizado e as unidades de } \\
\text { neurocirurgia nos Centros de Trauma } \\
\text { (CMT) precisam liderar o manejo } \\
\text { baseado em protocolo desses pacientes } \\
\text { em toda a sua rede. }\end{array}$ \\
\hline $\begin{array}{l}\text { La relación entre la } \\
\text { percepción del cuidador } \\
\text { sobre los síntomas } \\
\text { derivados del daño } \\
\text { cerebral en personas con } \\
\text { traumatismos } \\
\text { craneoencefálicos y su } \\
\text { propia Salud Mental }\end{array}$ & $\begin{array}{l}\text { Reyes-Aragón, } \\
\text { Landa, Romero, } \\
\text { e Arango- } \\
\text { Lasprilla, } \\
\text { (2015) }\end{array}$ & $\begin{array}{l}\text { Verificar se existe relação entre a } \\
\text { percepção do cuidador sobre os } \\
\text { sintomas apresentados pelo } \\
\text { paciente com traumatismo } \\
\text { cranioencefálico (TCE) e sua } \\
\text { saúde mental. }\end{array}$ & $\begin{array}{l}\text { Estudo } \\
\text { descritivo } \\
\text { com } \\
\text { abordagem } \\
\text { qualitativa }\end{array}$ & $\begin{array}{l}\text { Os achados no estudo sugerem a } \\
\text { necessidade de profissionais de saúde } \\
\text { de reabilitação desenvolverem e } \\
\text { implementarem intervenções } \\
\text { culturalmente apropriadas para reduzir } \\
\text { os sintomas neurocomportamentais em } \\
\text { pessoas com TCE, intervenções para } \\
\text { melhorar a autoestima, reduzir os } \\
\text { sintomas de depressão e sobrecarga em } \\
\text { cuidadores colombianos de indivíduos } \\
\text { com TCE. }\end{array}$ \\
\hline $\begin{array}{c}\text { Traumatismo } \\
\text { cranioencefálico em um } \\
\text { hospital-escola da cidade } \\
\text { de Campinas, São Paulo, } \\
\text { Brasil. }\end{array}$ & $\begin{array}{l}\text { Dalto e Escobar } \\
\qquad(2016)\end{array}$ & $\begin{array}{l}\text { realizar um estudo epidemiológico } \\
\text { sobre TCE no pronto-socorro de } \\
\text { um hospital-escola localizado no } \\
\text { distrito noroeste de saúde da } \\
\text { cidade de Campinas, auxiliando } \\
\text { assim na identificação de } \\
\text { propostas de resolução para os } \\
\text { problemas identificados }\end{array}$ & $\begin{array}{l}\text { Estudo } \\
\text { quantitativo }\end{array}$ & $\begin{array}{l}\text { Conclui-se, então, que há a necessidade } \\
\text { de criação de estratégias para } \\
\text { encaminhamento de tais pacientes a } \\
\text { outros destinos, não permanecendo os } \\
\text { mesmos internados em unidade de } \\
\text { pronto atendimento. }\end{array}$ \\
\hline $\begin{array}{l}\text { Underestimation of Pupil } \\
\text { Size by Critical Care and } \\
\text { Neurosurgical Nurses }\end{array}$ & $\begin{array}{l}\text { Kerr et al. } \\
\text { (2016) }\end{array}$ & $\begin{array}{c}\text { Avaliar as habilidades dos } \\
\text { enfermeiros para avaliar com } \\
\text { precisão o diâmetro da pupila e } \\
\text { detectar alterações pupilares em } \\
\text { pacientes com traumatismo } \\
\text { craniano. }\end{array}$ & $\begin{array}{l}\text { Estudo } \\
\text { descritivo, } \\
\text { com } \\
\text { abordagem } \\
\text { qualitativa }\end{array}$ & $\begin{array}{l}\text { Enfermeiros de cuidados intensivos e } \\
\text { neurocirúrgicos subestimaram o } \\
\text { tamanho da pupila, foram incapazes de } \\
\text { detectar anisocória e avaliaram } \\
\text { incorretamente a reatividade pupilar. O } \\
\text { uso padronizado de ferramentas de } \\
\text { avaliação da pupila, como o } \\
\text { pupilometro, é necessário para aumentar } \\
\text { a precisão e a consistência na medição } \\
\text { da pupila e, potencialmente, contribuir }\end{array}$ \\
\hline
\end{tabular}




\begin{tabular}{|c|c|c|c|c|}
\hline & & & & $\begin{array}{l}\text { para a detecção precoce de mudanças } \\
\text { sutis nas pupilas. }\end{array}$ \\
\hline $\begin{array}{l}\text { Diagramas para } \\
\text { interpretar y corregir } \\
\text { eventos fisiopatológicos } \\
\text { desencadenados tras el } \\
\text { traumatismo } \\
\text { craneoencefálico grave }\end{array}$ & $\begin{array}{l}\text { Godoy, Murillo- } \\
\text { Cabezas, egea- } \\
\text { Guerreiro, } \\
\text { Carmona-Suazo } \\
\text { e Munõz- } \\
\text { Sánchez } \\
\text { (2015) }\end{array}$ & $\begin{array}{l}\text { Realizar uma abordagem de } \\
\text { tratamento para traumatismo } \\
\text { cranioencefálico grave de acordo } \\
\text { com o padrão fisiopatológico. }\end{array}$ & $\begin{array}{l}\text { Estudo } \\
\text { descritivo, } \\
\text { com } \\
\text { abordagem } \\
\text { qualitativa }\end{array}$ & $\begin{array}{l}\text { Os diagramas expostos mostram de } \\
\text { forma simples o manejo desse tipo de } \\
\text { paciente, integrando as recomendações } \\
\text { da Brain Trauma Foundation, com as } \\
\text { informações disponíveis para otimizar a }_{\mathrm{ptiO}_{2}}\end{array}$ \\
\hline $\begin{array}{l}\text { The Effect } \\
\text { of Telenursing on } \\
\text { Referral Rates of Patients } \\
\text { With Head Trauma and } \\
\text { Their r Family's } \\
\text { Satisfaction } \\
\text { Discharge. After }\end{array}$ & $\begin{array}{l}\text { Shahrokhi, } \\
\text { Azimian, } \\
\text { Amouzegar e } \\
\text { Oveisi } \\
\text { (2018) }\end{array}$ & $\begin{array}{l}\text { Avaliar o efeito da telenfermagem } \\
\text { sobre as taxas de encaminhamento } \\
\text { de pacientes com traumatismo } \\
\text { cranioencefálico e a satisfação de } \\
\text { seus familiares após a alta. }\end{array}$ & $\begin{array}{l}\text { Estudo } \\
\text { descritivo, } \\
\text { com } \\
\text { abordagem } \\
\text { qualitativa }\end{array}$ & $\begin{array}{l}\text { O estudo aponta que os cuidadores } \\
\text { ficaram satisfeitos com o programa de } \\
\text { telenfermagem. Programas de } \\
\text { acompanhamento por meio de ligações } \\
\text { telefônicas foram eficazes e levariam a } \\
\text { maior satisfação do cuidador. }\end{array}$ \\
\hline
\end{tabular}

Fonte: Autores (2021).

Os estudos selecionados para análise apresentam resultados semelhantes, evidenciando a importância e a assistência prestada pela enfermagem diante da vítima de traumatismo cranioencefálico.

$\mathrm{O}$ alto índice de mortalidade em pacientes com TCE exige adoção de medidas que contribuam para a mudança desse quadro. Alguns estudos propõem a adoção de medidas preventivas para os fatores causais dos acidentes com veículos automotivos, agressões e quedas, como a obrigatoriedade do uso de equipamentos de proteção individual e campanhas de educação no trânsito, sendo este local um dos principais para a ocorrência deste problema (Carvalho, Silva, Viana, Madeira, Oliveira, \& Carvalho, 2020). Diante disso, mais da metade das mortes por TCE ocorrem no local do trauma, sem tempo hábil para reanimação. A abordagem inicial, a história clínica, o exame físico geral e a avaliação neurológica fornecem informações básicas para estratificação de risco de um paciente ter ou desenvolver lesão neurocirúrgica. Se realizadas de forma rápida e ordenadas, trarão grande benefício à vítima, evitando complicações (Oliveira, Pereira, \& Freitas, 2014).

A importância de investigar como aconteceu o acidente, pois conforme o mecanismo de lesão ou cinemática do trauma é possível predizer os tipos de danos e a gravidade a qual a vítima de trauma está exposta. (Caciano et al., 2019). Além disso, é importante destacar que o exame físico, preferencialmente, deve ser rápido e objetivo, uma vez que geralmente pacientes com TCE são politraumatizados e, portanto, adversidades como hipóxia, hipotensão, lesões instáveis da coluna vertebral devem ser procuradas e, convenientemente, tratados. O início de medidas terapêuticas para a estabilização das funções vitais nesse momento é essencial, deve-se prevenir e tratar a hipotensão e hipóxia, pois se acredita que apenas dez minutos de um desses sintomas sejam suficientes para provocar lesão cerebral secundária e piora do prognóstico (Rezer, Pereira, \& Faustino, 2020). Nesse sentido, as atividades realizadas pelo enfermeiro durante a assistência de urgência, devem priorizar a anamnese como componente essencial para a organização do processo assistencial e na conduta assumida pelos profissionais enfermeiros (Marinho, Santos, Filho, Valença, Santos, \& Júnior, 2019).

Além destes, o atendimento inicial as vítimas de TCE, realizado pelo enfermeiro é determinado pela realização da avaliação primária e, de acordo com o protocolo, o enfermeiro deve garantir permeabilidade das vias aéreas, a estabilização da coluna cervical, a utilização de oxigênio para uma ventilação adequada à vítima, monitorar a circulação e avaliação precoce da Escala de Coma de Glasgow (ECG). Em sequência realizar avaliação secundária, avaliando a reação pupilar, aferir os sinais vitais, exame físico da cabeça e coluna e repetição seriada da ECG (Oliveira, Pereira, \& Freitas, 2014).

Para a realização de um atendimento adequado, é essencial a utilização de equipamentos presentes, estrutura física adequada nas unidades de atendimento, recursos humanos, agilidade (Pulhrn, Westmoreland, \& McMahon, 2016).

A utilização de tecnologias digitais também é um importante recurso no contexto do cuidado de enfermagem atual, 
principalmente na adesão à aplicativos para avaliação de ECG, pois além de fornecer subsídios para o profissional na chegada da vítima com TCE à unidade hospitalar, proporciona possibilidade de aprendizado em outros momentos e locais, de forma individual e/ou coletiva (Reyes-Aragón, Landa, Romero, \& Arango-Lasprilla, 2015).

Uma abordagem humanizada e eficiente é indispensável no acolhimento primordial ao paciente em situação de emergência. Por isso, o profissional de enfermagem torna-se protagonista no cuidado ao cliente com TCE. A Sistematização da Assistência de Enfermagem, enquanto fator organizacional é garantidor no oferecimento de subsídios para o desenvolvimento de métodos e metodologias interdisciplinares, humanizadas e eficazes de cuidador (Dalto, \& Escobar, 2016).

A avaliação neurológica no paciente com trauma craniano deve receber uma atenção especial, principalmente na avaliação secundária, após se realizar o XABCDE, preconizado pelo ATLS, que padroniza o atendimento inicial ao paciente politraumatizado, para definir as prioridades na abordagem ao trauma. Ou seja, é uma forma rápida e fácil de memorizar todos os passos que devem ser seguidos com o paciente em politrauma. (Kerr et al., 2016). Durante a realização da avaliação primária, deve-se avaliar a ECG, já na admissão, avaliação dos padrões pupilares, avaliação de déficit motor e dos reflexos. A avaliação do nível de consciência do paciente pela ECG é a forma mais prática de se avaliar com objetividade o seu nível de consciência, mostrando o prognóstico com relação a sua lesão cerebral. A ECG avalia através do examinador a abertura ocular, melhor resposta verbal e melhor resposta motora, sendo atribuídos valores para cada achado no paciente (Godoy, MurilloCabezas, Egea-Guerreiro, Carmona-Suazo, \& Munõz-Sánchez, 2015).

O enfermeiro é o elemento chave da equipe responsável pelo atendimento ao paciente vítima de trauma durante cada fase do cuidado prestado. Sendo assim, ele deve buscar continuamente seu aprimoramento em relação às habilidades de liderança e ao mesmo tempo se atualizar nos moldes estabelecidos pelos programas educativos específicos para atuação nesta área de atendimento (Shahrokhi, Azimian, Amouzegar, \& Oveisi, 2018).

\section{Considerações Finais}

Conclui-se que o enfermeiro tem um papel fundamental no cuidado oferecido as vítimas de traumatismo cranioencefálico, sendo necessário que ele esteja apto para obter uma breve história do paciente, realize o exame físico, e execute o tratamento imediato, preocupando-se com a manutenção da vida. Deve aliar sua fundamentação teórica à capacidade de liderança, iniciativa e habilidades assistenciais e de ensino. Precisa ter raciocínio rápido, pois é responsável pela coordenação de uma equipe de enfermagem, sendo parte vital e integrante da equipe de emergência.

Desse modo, é perceptível que o atendimento de qualidade prestado pelo enfermeiro em tempo hábil ao paciente com traumatismo cranioencefálico minimiza gradativamente a incidência de possíveis sequelas ou o agravo das mesmas. Devendo este profissional ter conhecimentos prático e teórico atualizado para realizar uma avaliação rápida baseada em protocolos relacionados de TCE com o intuito de promover cuidados qualificados de enfermagem.

No entanto, a literatura científica mostra que são escassos os estudos referentes ao TCE, uma vez que, na prática profissional do enfermeiro, é comum este tipo de problema no ambiente hospitalar. Assim, é imprescindível o desenvolvimento de estudos e pesquisa sobre a temática do atendimento de enfermagem ao paciente vítima de TCE. Espera-se que esta revisão possa contribuir para a produção de conhecimentos, possibilitando uma melhor atenção em na prática de cuidado ao paciente com traumatismos cranioencefálicos, qualificando a assistência e oferecendo suporte social às famílias dos pacientes.

\section{Referências}

Caciano, K. R. P. S. et al. (2019). Intervenções de enfermagem para pacientes neurocríticos. Rev. Enferm. UFPE on line., 14, e243847.

Carvalho, O. N., Silva, I. M. C., Viana, M. R. P., Madeira, M. Z. A., Oliveira, A. D. S., \& Carvalho, A. R. B. (2020). Trauma cranioencefálico: perfil dos pacientes atendidos em um hospital público de Teresina. R. Pesq.: Cuid. Fundam._12, 946-952. 
Cunha, A. N. C., Araújo, L. M., \& Vieira, M. I. A. C. (2015). Atuação do enfermeiro a vítima de traumatismo cranioencefálico. Rev. El. Fac. Ceres., 4, 1-16.

Dalto, S. G., \& Escobar, E. (2016). Traumatismo cranioencefálico em um hospital-escola da Cidade de Campinas, São Paulo, Brasil. Enfer. Rev, 19 , 1-7.

Fettermann, F. A., Aranda, A., \& Kist, R. L. (2018). O atendimento de enfermagem a vítimas de trauma-craniocefálico. Rev. Elet. Ac. Saúde, 12 , $1270-1276$.

Godoy, D. A., Murillo-Cabezas, F., Egea-Guerreiro, J. J., Carmona-Suazo, J. A., \& Munõz-Sánchez, M.A. (2015). Diagramas para interpretar e resolver eventos fisiopatológicos desencadeados após lesão cerebral traumática grave. Med. Int., 39, 445-447.

Kerr, R. G. et al. (2016). Subestimação do tamanho da pupila por enfermeiros de cuidados intensivos e neurocirúrgicos. Am J Crit Care, $25,213-219$.

Marinho, C. S. R., Santos, J. N. A., Filho, L. A. M., Valença, C. N., Santos, E. G. O., \& Júnior, O. G. B. (2019). Accidente de tráfico: análisis de los casos de traumatismo cranoencefálico. Enferm. glob. 18

Mendes, K. D. S., Silveira, R. C. C. P., \& Galvão, C. M. (2008). Revisão integrativa: método de pesquisa para a incorporação de evidências na saúde e na enfermagem. Texto \& Contexto - Enfermagem 17(4758-764.

Monteiro, L. F., Frasson, M. Z., Wrsesinski, A., Bardini, A. V. L. S., Lin, J., \& Fernandes, A. F. Caracterização dos pacientes com trauma cranioencefálico grave admitidos em um hospital terciário. Arq. Catarin Med., 45. 02-16.

Oliveira, D. M. P., Pereira, C. U., \& Freitas, Z. M. P. (2014). Escalas para avaliação do nível de consciência em trauma cranioencefálico e sua relevância para a prática de enfermagem em neurocirurgia. Arq. Bras. Neurocir., 33, 22-32.

Oliveira, L. A. M., Soares, Y. K. C., Noleto, L. C., Fontinele, A. V. C., Galvão, M. P. S. P., \& Souza, J. M. (2018). Assistência de enfermagem vítimas de traumatismo crânio encefálico: revisão integrativa. Rev. UNINGÁ, Maringá, 55, 33-46.

Pulhorn, H., Westmoreland, L., \& McMahon, C. (2016). O manejo de traumatismo cranioencefálico menor (GCS 15-13) em uma Rede de Trauma. British Journal of Neurosurgery 30, 536-540.

Reyes-aragon, C. J., Landa, L. O., Romero, A. C., \& Arango-Lasprilla, J. C. (2015). La relación entre la percepción del cuidador sobre los síntomas derivados del daño cerebral en personas con traumatismos craneoencefálicos y su propia Salud Mental. Enferm. glob., 14, $181-192$.

Rezer, F., Pereira, B. F. O., \& Faustino, W. R. (2020). Conhecimento de enfermeiros na abordagem à vítima de traumatismo cranioencefálico. Journal Health NPEPS. 5, 291-302.

Ribeiro, A. P. S., Freitas, I. C. S., Gregório, L. A. M., \& Silva, K. C. (2015). Assistência de enfermagem frente aos pacientes que sofreram traumatismo cranioencefálico por arma de fogo: uma revisão bibliográfica. Rev. Fafibe On-Line, Bebedouro SP, 8 (1): $287-299$.

Santos, J. C. Traumatismo cranioencefálico no Brasil: análise epidemiológica. Resap, 3, 1-13.

Shahrokhi, A., Azimian, J., Amouzegar, A., \& Oveisi, S. (2018). O efeito da telenfermagem nas taxas de encaminhamento de pacientes com traumatismo craniano e na satisfação de suas famílias após a alta. Journal of Trauma Nursing, 15, 248-253.

Silva, F. S., \& Filha, F. S. S. C. (2017). Trauma cranioencefálico como um problema de saúde pública: uma revisão integrativa da literatura. ReonFacema. 3, 389-395.

Silva, Z. A., Pio, T. M., \& Maia, L. F. S. (2019). Trauma cranioencefálico: intervenções do enfermeiro no atendimento pré-hospitalar. Revista Recien., 9, 4653.

Soares, C. B., Hoga, L. A. K., Peduzzi, M., Sangaleti, C., Yonekura, T., \& Silva, D. R. A. D. Integrative Review: Concepts And Methods Used In Nursing. Revista da Escola de Enfermagem da USP. 48, 335-345.

Werlang, S. L., Badke, M. R., Freitag, V. L., Silva, G. S., Federizzi, D. S., \& Ribeiro, M. V. (2017). Enfermagem na assistência ao traumatismo cranioencefálico em um hospital universitário. J Health Sci., 19, 177-82. 